\title{
Derecho de autor y bibliotecas digitales: en busca del equilibrio entre intereses contrapuestos
}

\author{
Digital libraries and copyright: in search of a balance between \\ competing interests
}

Juan Carlos FERNÁNDEZ-MOLINA'

\section{RESUMEN}

El desarrollo tecnológico ha afectado de forma directa a las normas sobre derecho de autor, que en los últimos años está siendo modificada tanto nacional como internacionalmente. En términos generales, estas reformas legales se han centrado más en los intereses de los propietarios de los derechos que en los de los usuarios, incluidos entre ellos, las bibliotecas y otras instituciones similares, lo que supone una ruptura del equilibrio necesario entre los intereses de ambos sectores. El instrumento que posee las leyes de derecho de autor, para conseguir dicho equilibrio, son las excepciones y limitaciones a los derechos, entre los que se encuentran los denominados "privilegios de las bibliotecas. El objetivo de este trabajo es analizar en qué situación se encuentran en la actualidad estas excepciones y limitaciones a los derechos de autor, para ver en qué condiciones deben mantenerse, ampliarse y modificarse a la luz de los cambios producidos por el desarrollo de las tecnologías de la información y de la comunicación.

Palabras clave: derecho de autor; bibliotecas; privilegios de las bibliotecas; entorno digital.

\begin{abstract}
Technological development has had a direct impact on copyright legislation, that in the last few years it is being modified at national and international levels. Generally speaking, these legal reforms have been more focused on copyright holders than on users interests, included libraries and similar institutions, what entails a breach of needed balance between the interests of both stakeholders. Exceptions and limitations to the rights, including the "library privileges", constitute the tool of copyright laws to get that balance. The objective of the present study is to analyze the current situation of copyright exceptions and limitations in order to discuss under what conditions should be maintained, expanded and modified, having regard to the changes caused by the development of information and communications technologies
\end{abstract}

Keywords: copyright; libraries; library privileges; digital environment.

\section{INTRODUCCIÓN}

Las tecnologías digitales han transformado radicalmente de cómo las obras con derecho de autor son creadas y difundidas, además de las formas en que las bibliotecas hacen disponibles las obras. Estas instituciones, al llevar a cabo su misión, han comenzado a adquirir e incorporar a sus colecciones grandes cantidades de obras digitales para asegurar su disponibilidad continua, para las generaciones futuras.

\footnotetext{
1 Profesor Titular, Facultad de Comunicación y Documentación, Universidad de Granada, Colegio Máximo de Cartuja, Granada, España. E-mail: <icfernan@ugr.es>.

Recebido em 18/12/2007 e aceito para publicação em 19/3/2008.
} 
Estos cambios tecnológicos también han afectado de forma directa las normas sobre derecho de autor, que en los últimos años están siendo modificadas, tanto en el ámbito internacional como en las diferentes leyes nacionales. En términos generales, estas reformas legales se han centrado más en los intereses de los propietarios de los derechos que en los de los usuarios, incluidos entre ellos, las bibliotecas y otras instituciones de carácter cultural cuyo objetivo es facilitar el acceso a la cultura y a la información por parte de los ciudadanos. Esto supone una evidente ruptura del necesario equilibrio entre los intereses de ambos sectores, centrándose en el primero de los objetivos básicos de las leyes de derecho de autor: fomentar la creación y difusión de obras intelectuales proporcionando apoyo al autor mediante un monopolio de la explotación de la obra, pero olvidándose del segundo y no menos importante: facilitar el acceso a las mismas para beneficio de la sociedad en su conjunto.

El instrumento que posee las leyes de derecho de autor para conseguir el equilibrio entre ambos objetivos son las excepciones y limitaciones a los derechos, esto es, aquellos casos en que las obras pueden ser utilizadas sin permiso del propietario, ya sea de forma gratuita o con algún sistema de pago $o$ remuneración. Algunas de estas excepciones y limitaciones a los derechos de autor benefician directamente a las bibliotecas e instituciones similares para que puedan cumplir su misión y, desgraciadamente, se han quedado obsoletas y no son adecuadas para afrontar los problemas propios de los medios digitales. En algunos casos, porque no se han modificado en las actuales reformas de las leyes, y en otros porque, aunque sí han sido objeto de modificación, lo han sido de forma equivocada o insuficiente.

El objetivo de este trabajo es analizar en qué situación se encuentran en la actualidad las excepciones y limitaciones a los derechos de autor que benefician a las bibliotecas e instituciones similares, esto es, en qué condiciones deben mantenerse, ampliarse y modificarse a la luz de los cambios producidos por el desarrollo de las tecnologías de la información y la comunicación. Para ello se comienza haciendo una aproximación a los denominados "privilegios de las bibliotecas", siguiendo con una revisión general de su realidad en el entorno digital, tanto en los países desarrollados como en los en desarrollo. Finalmente se hacen algunas recomendaciones acerca de cómo reformar las leyes de derecho de autor para hacer frente a estos cambios y asegurar que se mantenga un adecuado equilibrio entre los intereses de los creadores y otros propietarios de los derechos de autor, por un lado, y las bibliotecas y sus usuarios, por el otro.

\section{LOS PRIVILEGIOS DE LAS BIBLIOTECAS}

Las limitaciones y excepciones a los derechos de los autores son numerosas y variadas debido a las diferentes razones que las justifican, lo que permite agruparlas en cuatro categorías (Guibault, 2002): 1) la defensa de derechos fundamentales; 2) la salvaguardia de la competencia; 3) el interés público; y 4) las imperfecciones del mercado.

A la tercera de estas categorías pertenecen las limitaciones y excepciones que tienen mayor interés en nuestro contexto. La defensa del interés público, en concreto, la promoción de la educación, la cultura y la investigación, son la base de los denominados privilegios de bibliotecas e instituciones similares (museos, archivos, hemerotecas, etc.). Su razón de ser se encuentra en que las funciones típicas de cualquier biblioteca colección, preservación y difusión de la informaciónimplican habitualmente en la copia, la distribución y la comunicación pública de obras con derecho de autor, por lo que entran en conflicto con los derechos de los autores, de autorizar y/o recibir una remuneración por tales usos de sus obras. Los ejemplos de actividades habituales de una biblioteca que afectan a los derechos de autor son múltiples: préstamo de ejemplares de obras a los usuarios; consulta de las obras en las instalaciones de la biblioteca o a distancia a través de la red, interna o no; permitir que los usuarios hagan copias de las obras utilizando las máquinas apropiadas libremente disponibles (fotocopiadoras, lector de microformas, impresora...); copia o transmisión de obras pedidas individualmente mediante servicios de préstamo interbibliotecario; copia de obras para sustituir ejemplares deteriorados, perdidos, robados o en formatos obsoletos.

Estos denominados "privilegios de bibliotecas" son permitidos porque se considera que estas instituciones llevan a cabo funciones de preservación y difusión de la información que benefician a la sociedad en su conjunto y promueven el bien común. Por esta razón, están incluidos en la legislación de derecho de autor de numerosos países, especialmente en los países anglosajones y europeos. Sin embargo, no aparecen en las legislaciones de algunos países de América Latina, 
por ejemplo Brasil (1998). También hay diferencias significativas en cuanto a cómo se configuran estos privilegios en las respectivas legislaciones nacionales, fundamentalmente en lo que se refiere a qué actos están permitidos o no, si alguno de ellos lleva embutida una remuneración y cuáles son las instituciones que se pueden beneficiar. A este respecto, hay que señalar que la dimensión de interés público de las bibliotecas varía dependiendo del tipo de biblioteca, ya sea pública o privada, con ánimo o no de ganancia, de acceso general o restringido, etc. También en este caso hay diferencias notables entre unos países y otros, de manera que los anglosajones los suelen regularizar de forma amplia y detallada, en tanto que en las legislaciones nacionales de los países de tradición jurídica latinocontinental están definidos con menos detalles y habitualmente de forma menos generosa.

\section{SITUACIÓN EN EL ENTORNO DIGITAL}

Hay un importante margen de libertad para que cada país establezca en su legislación nacional cuáles son las limitaciones y excepciones a los derechos de autor y cómo se definen. Sin embargo, dado que las obras intelectuales pueden circular de un país a otro y forman parte del comercio internacional, todos tienen que cumplir lo establecido en los tratados internacionales sobre la materia. Es decir, independientemente de cual sea la razón para su inclusión, cualquiera de las limitaciones y excepciones tienen que respetar los requisitos mínimos establecidos en estos tratados, fundamentalmente, el denominado "test de los tres pasos", establecido por primera vez en el artículo 9.2 del Convenio de Berna (OMPI, 1971), reconocido posteriormente por el acuerdo ADPIC de la Organización Mundial del Comercio (OMC, 1994) y por el nuevo tratado de la Organización Mundial de la Propiedad Intelectual dedicado a los derechos de autor en el entorno digital (OMPI, 1996). Estos tres pasos o condiciones, de carácter acumulativo, son: a) en ciertos casos especiales, b) que no entren en conflicto con la explotación normal de la obra, c) que no perjudiquen injustificadamente los intereses legítimos del autor.

Centrándonos a partir de este momento en la situación provocada por el entorno digital, es, el ya mencionado nuevo tratado de la OMPI de 1996, el que constituye el punto de partida de las reformas de las leyes nacionales de derecho de autor. Evidentemente, no pasa de largo por el problema de las limitaciones y excepciones a los derechos de autor, sino que las reglamenta en su artículo 10. Además de repetir la fórmula de los tres pasos, incluye otros elementos de gran interés que merecen ser analizados. En concreto, es especialmente importante la idea de que había que mantener el nivel de exigencia en el control de las limitaciones y excepciones, pero sin permitir a su vez una preponderancia absoluta de los intereses de los propietarios de los derechos. El resultado de este compromiso es el moderado contenido del artículo 10, su "declaración concertada" (que lo complementa y ayuda en su interpretación) e incluso el propio preámbulo. Este último incluye una frase muy significativa: "la necesidad de mantener un equilibrio entre los derechos de los autores y los intereses del público en general, en particular en la educación, la investigación y el acceso a la información". Por su parte, la declaración concertada del artículo 10 resuelve el difícil debate acerca de si es posible crear nuevas limitaciones y excepciones a los derechos de autor adaptadas a la nueva realidad tecnológica, al establecer que los Estados pueden "aplicar y ampliar debidamente las limitaciones y excepciones al entorno digital" en sus leyes nacionales y que pueden "establecer nuevas excepciones y limitaciones que resulten adecuadas al entorno de red digital". Hay que tener en cuenta que los representantes de los propietarios de los derechos, basándose en la idea de que "lo digital es diferente", querían que las limitaciones y excepciones sólo siguieran siendo aplicables en el entorno analógico, no en el digital; es decir, solicitaban su reducción o prácticamente eliminación si se trataba de obras digitales. Afortunadamente, el texto final no accedió completamente a sus exigencias. En definitiva, el nuevo tratado de la OMPI no supone una disminución de las limitaciones y excepciones al derecho de autor, sino una simple adaptación a las nuevas circunstancias del entorno digital, algo totalmente lógico si llevamos en cuenta que las razones en las que se basan, en especial la defensa de derechos fundamentales y el interés público, son igualmente válidas para un entorno impreso o digital. Desgraciadamente, y como veremos a continuación, estas posibilidades no han sido debidamente aprovechadas por los países en las reformas de sus leyes nacionales de derecho de autor. En términos generales, no se han hecho las necesarias ampliaciones y/o adaptaciones de las limitaciones ya existentes, ni se han creado las nuevas limitaciones 
requeridas por los cambios tecnológicos, o se ha hecho de forma tan mezquina que los resultados son realmente pobres.

Si nos centramos en el ámbito europeo, los privilegios de las bibliotecas e instituciones similares aparecen incluidos en la directiva (Unión Europea, 2001) que tenía el objetivo de armonizar las leyes nacionales y adaptarlas a las exigencias del Tratado de la OMPI. En concreto, en el artículo 5.2.c, en lo que se refiere a las reproducciones, y en el 5.3.n, en lo relativo a la comunicación pública. El primero de ellos permite actos específicos de reproducción efectuados por bibliotecas accesibles al público y siempre que no tengan intención de obtener beneficio económico o comercial directo o indirecto. Lo más destacable de esta disposición es que da lo mismo si las bibliotecas son públicas o privadas, lo importante es que estén accesibles al público, y que no se exige remuneración compensatoria para los titulares de los derechos. Desgraciadamente, y en términos generales, su transposición a las legislaciones nacionales no ha sido excesivamente generosa. Por ejemplo, en la reforma de la ley española (España, 2006) se han incluido restricciones no exigidas por la directiva. Así, su artículo 37.1 establece que las reproducciones sólo son posibles con objetivos de investigación o conservación, pero no para estudio personal. De esta forma, si no se hace una interpretación amplia del término "investigación", sólo en aquellas bibliotecas en las que indiscutiblemente sus usuarios tienen fines de investigación pueden disfrutar de esta limitación. En cuanto a la conservación, hay que reseñar que en principio no aparecía en su redacción, pero finalmente fue incluida gracias al esfuerzo de FESABID (2005). Tampoco es muy positivo el contenido de la ley de Italia (2003), cuyo artículo 68 establece que las bibliotecas e instituciones similares pueden hacer fotocopias de las obras que componen su colección, siempre que lo hagan para sus propios servicios y sin ventaja económica o comercial directa o indirecta. También permite las reproducciones hechas en las bibliotecas para uso personal mediante medios reprográficos siempre que no se supere el 15\% de cada volumen o fascículo de una revista. Es decir, sólo permite fotocopias, no copias digitales. Más adecuada parece la legislación de Francia (2006), cuyo artículo 122 5(8) permite los actos de reproducción específicos efectuados por bibliotecas accesibles al público, museos o archivos que no busquen ventaja comercial o económica directa o indirecta. Al contrario que otra leyes, no hace referencia a los objetivos de tales reproducciones: investigación, preservación..., ni afortunadamente las limita a los medios reprográficos.

En cuanto al artículo 5.3.n, sin duda es el más interesante para el entorno digital, ya que se refiere al derecho de comunicación pública, esto es, el afectado por los actos de transmisión digital a través de las redes, ya sean internas o externas. Permite los actos de comunicación a personas concretas del público o la puesta a su disposición de las obras que componen su colección, para efectos de investigación o estudio personal, siempre que se haga a través de terminales especializados situados en sus instalaciones y dichas obras no sean objeto de condiciones de adquisición o de licencia. Sorprende que, al contrario que con las reproducciones, estos actos de comunicación pública estén limitados a fines de "investigación o de estudio personal". Además, esta limitación tiene más restricciones. Por un lado, sólo se pueden mostrar obras "de sus colecciones", lo que excluye por ejemplo a las obras conseguidas mediante préstamo interbibliotecario. Además, sólo se pueden consultar las obras que no sean objeto de condiciones de adquisición o licencia, lo que anula en buena medida la utilidad de esta excepción (Fernández-Molina, 2003). También exige que la comunicación se lleve a cabo a través de terminales especializados, lo que supone que no es posible que los usuarios se conecten a la red de la biblioteca con sus propios ordenadores. Por último, la comunicación debe llevarse a cabo en el edificio de la Biblioteca, lo que deja en el aire qué pasa con las bibliotecas virtuales o con los accesos remotos que muchas bibliotecas ofrecen a sus usuarios (por ejemplo, alumnos y profesores de universidades). También resulta decepcionante que no se prevea nada para la transmisión de obras a través de Internet, ya sea a otras instituciones o bibliotecas o a estudiantes o profesionales de otras instituciones (Garrote, 2001), es decir, no está incluido lo que podríamos denominar como préstamo inter-bibliotecario digital. Pese a estas innumerables restricciones, podemos encontrar algunas aplicaciones a esta excepción, entre ellas, la preservación de materiales (por ejemplo, digitalizar obras de difícil acceso o cuyo uso físico pueda deteriorarlas y dejar que los usuarios las consulten a través de los terminales que la biblioteca disponga (Riera, 2002).

Su transposición ha sido diversa, según los países. Por ejemplo, Italia se limita a reproducir de forma literal el texto de la directiva, pero España o Francia 
restringen aún más su contenido. Así, la ley española elimina el fin del estudio personal, de manera que esta comunicación sólo está permitida para fines de investigación; y, además, también incluye la obligación de remunerar al autor, exigencia que tampoco está reconocida en la directiva. Peor es el caso de Francia, ya que la transposición de este artículo de la directiva no se ha incluido en su código de propiedad intelectual, sino en el Código de Patrimonio (Francia, 2004), en concreto, en su artículo L 132-4, que establece que el autor no puede prohibir a los organismos depositarios la consulta de la obras por parte de investigadores acreditados dentro de sus instalaciones. También incluye la posibilidad de hacer una reproducción para facilitar la consulta in situ. Es evidente que esta transposición se ha quedado muy lejos de lo permitido por la directiva, ya que sólo afecta a aquellas escasísimas bibliotecas que les han concedido el depósito legal.

Este breve análisis de la directiva europea y de la transposición realizada por países que representan el modelo latino-continental (España, Francia e Italia) pone claramente de manifiesto que el desarrollo de lo establecido en el Tratado de Derecho de Autor de la OMPI de 1996 no ha sido muy adecuado, sino que por el contrario ha pecado de mezquino y restrictivo. La directiva no ha incluido algunas limitaciones imprescindibles para las bibliotecas actuales; y algunas de las legislaciones nacionales que la han desarrollado, incluso restringen aún más lo establecido en ella. Como consecuencia, las bibliotecas se encuentran con graves dificultades para seguir desempeñando sus funciones de forma satisfactoria si se trata de obras en formato digital, cada día más numerosas en sus colecciones.

A conclusiones similares llegamos si analizamos la ley del país que ha actuado como pionero en este campo: Estados Unidos (1998), en especial por su reglamentación de los sistemas que protegen tecnológicamente las obras con derechos de autor, los tristemente famosos DRMS (Digital Rights Management Systems), ya que si hay conflicto entre el disfrute de los privilegios de las bibliotecas y la protección prestada por estos sistemas, siempre se decide a favor de estos últimos. Por ejemplo, no podrá hacerse una copia permitida por la ley si para ello hay que pasar por encima del sistema que controla su acceso o uso. Tampoco la biblioteca podrá aprovechar las limitaciones que la benefician si el contrato (licencia) que regula el uso de recursos electrónicos se lo impide. Únicamente la ley de Australia (2000) merece una calificación positiva, aunque no suficiente para las verdaderas necesidades de las bibliotecas en la actualidad. Como aspectos positivos hay que señalar que permite reproducir obras digitalmente para propósitos "administrativos", o hacerlas para objetivos de preservación; o incluso es posible que una biblioteca copie o envíe a otra un artículo electrónico o una parte de una obra, ya sea para incluirla en su colección o para proporcionársela a un usuario, en ambos casos, sólo si la obra no está disponible comercialmente. Esta ley ha sido recientemente reformada (Australia, 2006), manteniendo estas excepciones e incluso ampliándolas. Por ejemplo, ahora también pueden acogerse a ellas las bibliotecas comerciales, siempre que su colección esté accesible al público. No obstante, aún siendo generosa con las bibliotecas, esta ley mantiene el problema de que todas estas limitaciones y excepciones pueden quedar anuladas por los contratos o licencias de los recursos en línea.

Si analizamos ahora la situación de los países emergentes y en desarrollo, en concreto los de América Latina, nos encontramos con una situación mucho más negativa. Así, en muchos de ellos estos privilegios de las bibliotecas ni siquiera están reconocidos en sus legislaciones de derecho de autor, por ejemplo Brasil, Chile o Argentina, lo que deja a sus bibliotecas en la extraña situación de que buena parte de sus actividades habituales son -al menos en teoría- ilegales. En aquellos otros países en que sí se reconocen, por ejemplo Colombia (1982), México (2003), Paraguay (1998), Perú (1996) o Venezuela (2003), su contenido es pobre y escaso, ya que normalmente sólo permiten hacer copias por razones de preservación o conservación, sin llegar más allá ni amparar otro tipo de actividades bibliotecarias. Además, ninguna de ellas ha sido modificada para hacer frente a los problemas del entorno digital, por lo que su contenido es ya claramente obsoleto.

A este respecto, merece la pena comentar, al menos brevemente, que los tratados internacionales (de la OMPI y de la OMC) y los acuerdos bilaterales de libre comercio que se han aprobado en la última década han provocado una disminución del acceso al conocimiento, en especial en los países en desarrollo, ya que el contenido de dichos acuerdos y tratados favorece de forma clara a los intereses de los países más ricos (Norteamérica y la Unión Europea), que son exportadores netos de productos con derechos de autor. Si las leyes de derecho de autor no son adaptadas al 
nivel de desarrollo de cada uno de los países, ¿̇cómo va a ser posible que las naciones más pobres progresen en el analfabetismo a la alfabetización?, ¿̇cómo van a pasar del estatus de "en desarrollo" a "desarrollado"? (Hackett, 2005). Una solución a estos problemas puede venir de iniciativas tales como el proyecto de Tratado de Acceso al Conocimiento (A2K, 2005), promovido por Brasil y Argentina, y la denominada Agenda del Desarrollo de la Organización Mundial de la Propiedad Intelectual, propuesta también por iniciativa de Brasil y Argentina (OMPI, 2004), y que tras varios años de discusiones ha sido finalmente aprobada el pasado mes de octubre (OMPI, 2007).

\section{CÓMO DEBERÍAN REGULARIZARSE}

Estas insuficiencias e inadecuaciones en las nuevas leyes de derecho de autor han sido detectadas y estudiadas tanto por las asociaciones de bibliotecarios como por diferentes comités de expertos, especialmente en los países anglosajones (Australia Senate, 2003; Canadian Library Association, 2006; Library of Congress, 2005), dando lugar a informes, declaraciones o recomendaciones. Especialmente interesantes son los principios establecidos por la Library Copyright Alliance (2004), ya que se centra específicamente en los problemas de los países en vías de desarrollo.

Aunque, como ya hemos comentado, cada ley y situación nacional tiene sus particularidades, hay una serie de principios y necesidades de carácter general que son aplicables a prácticamente cualquier país y legislación. A continuación vamos a comentar brevemente aquellos que consideramos más importantes.

Uno de los principios básicos que deben guiar la legislación de derecho de autor es el de neutralidad tecnológica. Esta neutralidad garantiza que tanto para los propietarios de los derechos como para los usuarios se aplican igual los conceptos de la ley de derecho de autor, independientemente de cuál sea el formato de la obra, analógico o digital. El resultado es un equilibrio justo entre los intereses de los propietarios y los usuarios, más allá de los formatos de la obra. También supone que la ley es aplicable igualmente a una variedad de circunstancias. En definitivo, esta neutralidad aporta coherencia y hace predecible la aplicación de la ley en el entorno digital. Desgraciadamente, ese principio se está erosionando rápidamente; por ejemplo, es habitual que las leyes establezcan (la directiva europea, entre otras) que la consulta y comunicación pública de las obras no es posible fuera de las instalaciones o locales de la biblioteca, es decir los usuarios remotos no tienen este derecho, sólo los que estén físicamente en la biblioteca (Lipinski, 2003).

Uno de los pilares en los que se basan estos privilegios es que no todas las bibliotecas o instituciones similares pueden beneficiarse de ellos. Normalmente se exige que sean de carácter público o, en el caso de que sean privadas, es necesario que su acceso sea público. Pues bien, en las nuevas leyes los criterios para que una biblioteca pueda beneficiarse de esta limitación a los derechos de autor son excesivamente restrictivos y destilan una filosofía claramente "predigital". Por ejemplo, se suele solicitar que sean bibliotecas "físicas", no virtuales, o se establece que el acceso sólo está permitido dentro de las instalaciones físicas de la biblioteca. Esto es un disparate si llevamos en consideración de que hay un imparable proceso hacia el caracter virtual y nó físico de las bibliotecas y de las formas de acceso por parte de sus usuarios. Estos absurdos y anacrónicos requisitos son un claro reflejo del no cumplimiento del principio anteriormente comentado, el de la neutralidad tecnológica.

Algo similar sucede con la enseñanza de carácter virtual o no presencial, seriamente dificultada con las restricciones impuestas por la legislación actual. Parece claro que tanto los educadores como los alumnos a distancia digitales no deberían tener menos derechos ni afrontar un mayor nivel de responsabilidad para asegurar los derechos de autor que los que tenían en el entorno no digital. Sin una legislación más generosa, las bibliotecas ligadas a instituciones que imparten educación digital, difícilmente pueden llevar a cabo su función

Hay que garantizar que las limitaciones y excepciones a los derechos de autor no queden sin efecto debido a la protección tecnológica. Las nuevas normas incluidas en las leyes de derecho de autor, para impedir la elucidación de la protección tecnológica de las obras, suponen que al propietario de los derechos se les permite poner una valla alrededor de un espacio público (al impedir también los usos no infractores). En definitiva, estas disposiciones dan al propietario de los derechos, un derecho legal al imposibilitar tales usos permitidos, de manera que el control tecnológico se impone sobre los derechos que la ley concede a las bibliotecas y sus usuarios. Es esencial que las limitaciones y excepciones establecidas en la ley de derecho de autor relacionadas con la gestión y mantenimiento de las colecciones y los actos realizados 
en representación de los usuarios incluidos dentro de tales limitaciones, continúen siendo válidos incluso, si es necesario, eludir las medidas tecnológicas usadas por los propietarios de los derechos de autor (FernándezMolina, 2003).

Un problema parecido es el planteado por las licencias de recursos electrónicos. En la actualidad, la tradicional venta de las publicaciones está siendo sustituida por estos contratos, firmados por el proveedor de la información y la biblioteca, en los que se establecen las condiciones de uso de tales recursos (revistas digitales, e-books, bases de datos...). Pues bien, de manera habitual estas licencias suelen prohibir disfrutar de las limitaciones a los derechos que benefician a las bibliotecas, convirtiéndolas en auténtico papel mojado. Es necesario que la nueva legislación asegure que estas nuevas técnicas comerciales sean compatibles con las limitaciones y excepciones a los derechos de autor, estableciendo que las más importantes de éstas (las que benefician a las bibliotecas y sus usuarios, por ejemplo) tengan carácter imperativo y no puedan ser anuladas a través de contratos (Fernández-Molina, 2004). Desgraciadamente, en prácticamente ninguna de las nuevas leyes se ha conseguido ésto hasta ahora, aunque lo han solicitado las asociaciones de bibliotecarios de varios países. Si se unen estos dos últimos problemas, protección tecnológica y contractual, nos encontramos con un peligroso proceso de privatización del acceso a la información, dejando en manos de los propietarios de los derechos quién, cuándo y bajo qué condiciones es posible acceder a la información, independientemente de lo establecido por la ley (Elkin-Koren, 2000).

Otro problema de las bibliotecas actuales, que no soluciona la actual legislación de derecho de autor, es el de las reproducciones y comunicaciones necesarias para la preservación digital. Las bibliotecas necesitan "refrescar" o emigrar los contenidos para hacer frente a la evolución y disponibilidad de la tecnología actual y no tener que esperar a que los formatos más viejos estén obsoletos. Algunas leyes permiten algunas reproducciones por razones de preservación, pero con excesivas restricciones y no de forma preventiva, por lo que en muchas ocasiones ya es demasiado tarde cuando legalmente se puede actuar (Ayre; Muir, 2004). También en este caso las normas parecen haber sido pensadas exclusivamente para el mundo analógico, no para el digital (Fernández-Molina; Guimarães, 2007).

Por último, parece claro que determinados sitios web también merecen ser preservados, por lo que debe crearse una limitación a los derechos de autor que permita la captura y preservación de determinados sitios y contenidos web, estableciendo además qué bibliotecas en concreto podrán ser las beneficiadas.

\section{CONCLUSIONES}

Las posibilidades legales ofrecidas por el Tratado de Derecho de Autor de la OMPI para ampliar y adaptar las limitaciones y excepciones al nuevo entorno tecnológico no han sido aprovechadas. Incluso aquellas leyes recientemente reformadas, siguen lo que podríamos denominar como una filosofía "pre-digital". Si la legislación no es modificada de forma adecuada las bibliotecas no podrán seguir cumpliendo su labor, ya que sólo podrán hacerlo con sus fondos y recursos no digitales, cada vez más minoritarios.

Las bibliotecas no son una amenaza para los derechos de autor. Por el contrario, son usuarios responsables de los materiales con derecho de autor, y se esfuerzan en educar a sus usuarios en su utilización legal y apropiada para la educación, la investigación y el trabajo. Desgraciadamente, ni los propietarios de los derechos ni el legislador de la mayoría de los países parecen estar conscientes de ello, por lo que las nuevas leyes imponen excesivas y absurdas restricciones a las bibliotecas impidiéndoles desarrollar adecuadamente su función social de facilitar el acceso de los ciudadanos a la información. Esta ausencia de equilibrio está produciendo que la legislación de derecho de autor vaya en contra de las bibliotecas, del aprendizaje, de las personas discapacitadas, del acceso al conocimiento $y$, en definitiva, contra el desarrollo.

Si en los países desarrollados resulta muy dudosa la conveniencia de una excesiva protección de los derechos de autor, es evidente su inadecuación para los países en desarrollo, dado que son importadores de productos con derechos de autor, no exportadores. Además, no cuentan con infraestructuras científicas y tecnológicas suficientemente sofisticadas como para sacar partido de la protección. Por lo tanto, estos países deberían tener unos sistemas de derechos de autor menos proteccionistas y más adaptados a sus circunstancias concretas. A este respecto, hay que reseñar el éxito inicial obtenido por los países promotores de la Agenda para el Desarrollo de la OMPI, recientememente aprobada, que supone un excelente punto de partida para que los intereses de los países en desarrollo sean llevados en cuenta a partir de ahora. 
AUSTRALIA. Copyright Amendment (Digital Agenda) Act 2000. Disponível em: <http://scaletext.law.gov.au/html/comact/10/ 6223/top.htm>. Acesso em: 10 dic. 2007.

AUSTRALIA. Copyright Amendment Act 2006. Disponível em: $<w w w$. copyright.org.au/pdf/acc/infosheets_pdf/g096.pdf> . Acesso em: 10 dic. 2007.

AUSTRALIA. Senate. Libraries in the online environment. 2003. Disponível em: <http://www.aph.gov.au/Senate/committee/ ecita_cte/completed_inquiries/2002-04/online_libraries/report/ report.pdf>. Acesso em: 10 dic. 2007.

AYRE, C.; MUIR, A. Right to preserve?: The copyright and licensing for digital preservation project. Final report. Loughborough University, 2004. Disponível em: <https://dspace.lboro.ac.uk/ dspace/bitstream/2134/343/1/Final\%20report.pdf>. Acesso em: 10 dic. 2007

A2K. Treaty on access to knowledge (Draft 9 May 2005). Disponível em: <http://www.cptech.org/a2k/a2k treaty may9.pdf>. Acesso em: 10 dic. 2007.

BRASIL. Lei n 0.610 , de 19 de fevereiro de 1998. Altera, atualiza e consolida a legislação sobre direitos autorais e dá outras providencias. Disponível em: <http://www.planalto.gov.br/ccivil_03/ Leis/L9610.htm>. Acesso em: 10 dez. 2007.

CANADIAN LIBRARY ASSOCIATION. Protecting the public interest: information for the Canadian library and information community on Bill C-60, An act to amend the Copyright Act, 2006. Disponível em: <http://www.cla.ca/resources/ protecting the public interest.pdf>. Acesso em: 10 dic. 2007.

COLOMBIA. Ley 23 de 1982 sobre Derechos de Autor. Disponível em: <http://www.cerlalc.org/derechoenlinea/dar/ leyes_reglamentos/Colombia/Ley_23.htm $>$. Acesso em: 10 dic. 2007.

ELKIN-KOREN, N. The privatization of information policy. Ethics and Information Technology, v.2, n.4, p.201-209. 2000.

ESPAÑA. Ley 23/2006, de 7 de Julio, por la que se modifica el texto refundido de la Ley de Propiedad Intelectual, aprobado por el Real Decreto Legislativo 1/1996, de 12 de abril. Disponível em: <http://www.boe.es/boe/dias/2006/07/08/pdfs/A2556125572.pdf> . Acesso em: 10 dic. 2007.

ESTADOS UNIDOS. Digital Millennium Copyright Act 1998. Disponível em: http://www.copyright.gov/legislation/hr2281.pdf>. Acesso em: 10 dic. 2007.

FERNÁNDEZ-MOLINA, J.C. Laws against the circumvention of copyright technological protection. Journal of Documentation, v.59, n. 1, p.41-68. 2003.

FERNÁNDEZ-MOLINA, J.C. Licensing agreements for information resources and copyright limitations and exceptions. Journal of Information Science, v.30, n.4, p.337-346. 2004.
FERNÁNDEZ-MOLINA, J.C.; GUIMARÃES, J.A.C. (2007). Las nuevas leyes de derecho de autor: Żadecuadas para la preservación digital? Information Research, v. 12, n.4, paper 322. 2007. Disponível em: http://InformationR.net/ir/12-4/paper322.html> Acesso em: 10 dic. 2007.

FESABID. Alegaciones al Proyecto de Ley, de 26 de agosto de 2005, de modificación del texto refundido de la Ley de Propiedad Intelectual. 2005. Disponível em: <http://www.fesabid.org/ federacion/gtrabajo/bpi/alegacionesfesabidseptiembre2005.pdf > . Acesso em: 10 dic. 2007.

FRANCIA. Code du patrimoine (Ordonnance $n^{\circ} 2004-178 d u$ 20 février 2004 relative à la partie législative du code $d u$ patrimoine). Disponível em: <http://www.legifrance.gouv.fr/ WAspad/UnTexteDeJorf? numjo $=$ MCCX0300157R > . Acesso em: 10 dic. 2007.

FRANCIA. Loi no 2006-961 du 1 er août 2006 relative au droit d'auteur et aux droits voisins dans la société de l'information. Disponível em: <http://www.legifrance.gouv.fr/imagesJOE/2006/ 0803/joe_20060803_0178_0001.pdf>.Acesso em: 10 dic. 2007.

GARROTE, I. El derecho de autor en Internet: la directiva sobre derechos de autor y derechos afines en la sociedad de la información. Granada: Comares. 2001.

GUIBAULT, L.M.C.R. Copyright limitations and contracts: an analysis of the contractual overridability of limitations on copyright. The Hague, Kluwer Law International. 2002.

HACKETT, T. Learning with libraries and copyright issues. In: INFORMATION MEETING ON EDUCATIONAL CONTENT AND COPYRIGHT IN THE DIGITAL AGE IN CONJUNCTION WITH THE THIRTEENTH SESSION OF THE WIPO STANDING COMMITTEE ON COPYRIGHT AND RELATED RIGHTS (SCCR), 2005. Disponível em: < www.wipo.int/edocs/mdocs/copyright/ en/educ cr im 05/educ cr im 05 www 53634.pdf>. Acesso em: 10 dic. $200 \overline{7}$

ITALIA. Decreto Legislativo 9 aprile 2003, n. 68 "Attuazione della direttiva 2001/29/CE sull'armonizzazione di taluni aspetti del diritto d'autore e dei diritti connessi nella società dell'informazione". Disponível em: < http://www.parlamento.it/parlam/leggi/deleghe/ 03068dl.htm>. Acesso em: 10 dic. 2007.

LIBRARY COPYRIGHT ALLIANCE. Library-related principles for the International Development Agenda of the World Intellectual Property Organization. 2004. Disponível em: <http:// www.librarycopyrightalliance.org/wipo.htm $>$. Acesso em: 10 dic. 2007.

LIBRARY OF CONGRESS. The Section 108 Study Group. 2005. Disponível em: <http://www.loc.gov/section 108/index.html>. Acesso em: 10 dic. 2007.

LIPINSKI, T.A. The myth of technological neutrality in copyright and the rights of institutional users: recent legal challenges to the information organization as mediator and the impact of the 
DMCA, WIPO, and TEACH. Journal of the American Society for Information Science and Technology, v.54, n.9, p.824-835. 2003.

MÉXICO. Ley Federal del Derecho de Autor. 2003. Disponível em: <http://www.cerlalc.org/derechoenlinea/dar/ leyes_reglamentos/Mexico/Ley_Federal.htm $>$. Acesso em: 10 dic. 2007.

OMC. Acuerdo sobre los aspectos de los derechos de propiedad intelectual relacionados con el comercio. Anexo 1C del Acuerdo de Marrakech por el que se establece la Organización Mundial del Comercio de 15 de abril de 1994. Disponível em: <http:// www.wto.org/spanish/docs_s/legal_s/27-trips.pdf $>$. Acesso em: 10 dic. 2007

OMPI. Convenio de Berna para la protección de las obras literarias y artísticas (Acta de París del 24 de julio de 1971 y enmendado el 28 de septiembre de 1979). Disponível em: <http://www.wipo.int/ treaties/es/ip/berne/trtdocs wo001.html>. Acesso em: 10 dic. 2007.

OMPI. Tratado de la OMPI sobre Derecho de Autor. 1996. Disponível em: <http://www.wipo.int/treaties/es/ip/wct/ trtdocs wo033.html>. Acesso em: 10 dic. 2007.

OMPI . Propuesta de Argentina y Brasil para establecer un programa de la OMPI para el desarrollo (Asamblea General de la OMPI, Ginebra, 27 septiembre a 5 octubre de 2004). Disponível em: <http://www.wipo.int/documents/es/document/govbody/ wo_gb_ga/pdf/wo_ga_31_11.pdf>. Acesso em: 10 dic. 2007.

OMPI. Informe general (Asambleas de los Estados miembros de la OMPI, Ginebra, 24 de septiembre a 3 de octubre de 2007),
Disponível em: <http://www.wipo.int/edocs/mdocs/govbody/es/ a_43/a_43_16-main 1.pdf>. Acesso em: 10 dic. 2007.

PARAGUAY (1998). Ley de Derecho de Autory Derechos Conexos (Ley $n^{\circ}$ 1328). 1998. Disponível em: <Hp://www.cerlalc.org/ derechoenlinea/dar/leyes reglamentos/Paraguay/ Ley_1328.htm>. Acesso em: 10 dic. 2007.

PERÚ. Ley sobre el Derecho de Autor, Decreto Legislativo no 822 de 1996. Disponível em: <http://www.cerlalc.org/ derechoenlinea/dar/leyes reglamentos/Peru/Ley $822 . \mathrm{htm}>$. Acesso em: 10 dic. 2007.

RIERA, P. Posibles consecuencias de la transposición de la Directiva 2001/29/CE para las bibliotecas. In: PROCEEDINGS CONTENIDOS Y ASPECTOS LEGALES EN LA SOCIEDAD DE LA INFORMACIÓN (CALSI), Valencia (Spain), 2002. Disponível em: <eprints.rclis.org/archive/00000505/01/ 05 Patricia Riera Derechos de Autor.pdf>. Acesso em: 10 dic. 2007.

UNIÓN EUROPEA. Directiva 2001/29/CE del Parlamento Europeo y del Consejo de 22 de mayo de 2001 relativa a la armonización de determinados aspectos de los derechos de autor y derechos afines en la sociedad de la información, DOCE 22.6.2001, L167

VENEZUELA. Ley sobre el Derecho de Autor. 1993. Disponível em: <http://www.cerlalc.org/derechoenlinea/dar/ leyes_reglamentos/Nenezuela/Ley_derautor.htm $>$. Acesso em: 10 dic. 2007.
131

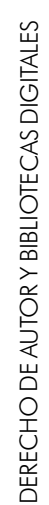


